\title{
Taking into account heat losses in front-face pulse IR thermography experiment for thermal diffusivity identification
}

\author{
by D.Balageas* and J.-M. Roche** \\ * ENSAM ParisTech, Institute of Mechanics and Engineering of Bordeaux (I2M), TREFLE Dept., 33305 Talence \\ cedex, France, daniel.balageas@wanadoo.fr \\ ** ONERA (The French Aerospace Lab), Materials and Structures Dept., BP 72, 92322 Châtillon cedex, France, \\ jean-michel.roche@onera.fr
}

\begin{abstract}
Thermal diffusivity can be measured by analysing the pulse response of the rear-face or of the front-face using a single detector or an IR camera. For the front-face configuration a simple identification method is proposed in the case of significant heat losses. The method is based on original correlations established between the occurrence times and values of the first and second logarithmic derivatives obtained by the thermographic signal reconstruction (TSR) method. The intrinsic accuracy of the identified diffusivity is evaluated by numerical simulations and validated by the analysis of experimental thermograms. Guidelines are proposed to optimise the identification procedure.
\end{abstract}

\section{Introduction}

Pulsed photothermal radiometry and stimulated IR thermography, studied since several decades are mainly developed for NDE and thermophysical characterisation purposes. For them, several data processing methods exists since a long time or are still in development. Most of the developments, and the present work in particular, are focused on the single-sided (or front-face) technique in which stimulation and thermal monitoring are on the same side of the studied object. Contrarily to what has been done for the rear-face technique [1-5], up to now, for the front-face technique, attention has been rarely paid to the heat losses which take place during the cooling phase following the stimulation [68]. This is not a problem when the conditions (pressure and temperature) are near of the ambient, the structures are made of good heat conductors and their thickness small or moderate. Nevertheless, these phenomena limit the field of application of the technique, in particular when dealing with the metrology of the diffusivity. In this context, the present work aims to take into account the heat losses phenomena in the identification procedure of this parameter, in a way similar to what has been done by many researchers in the past for the rear-face technique. The chosen approach consists to select a few critical points of the thermogram (temperature increase time history) to achieve the diffusivity or thickness identification. Such simple approach, not so sophisticated as inverse methods, remains interesting thanks to its rapidity and simplicity.

After a recall of pulse stimulated radiometric techniques and of the heat losses influence, the principle of the present approach, derived from the thermographic signal reconstruction (TSR) method is described, showing how to identify in presence of heat losses the diffusivity from the values and the occurrence times of the maximums of the first and second logarithmic derivatives of the thermogram fitted by the TSR method. The intrinsic accuracy is evaluated and finally the validation is obtained by applying the method to the characterisation of a coupon of carbon-epoxy composite.

\section{How heat losses disturb the pulsed thermograms}

Figure 1 presents the normalised adiabatic thermograms of the two faces of a plate submitted to a pulse heating. They are obtained by dividing the temperatures by the final asymptotic value. The normalised time is the Fourier number: $\mathrm{Fo}_{\mathrm{o}} \mathrm{kt} / \mathrm{L} 2$, where $\mathrm{t}$ is the time, $\mathrm{L}$ the thickness of the coupon and $\mathrm{k}$ its diffusivity. The thermograms are presented for two values $(0$ and 1$)$ of the normalised heat transfer coefficient at the wall, the Biot number: $B i=h L / k$, with $h$ the linear heat transfer coefficient supposed identical for both faces and $k$ the thermal conductivity). These results are for 1-D configuration.

The front-face normalised thermogram renders a cooling of decreasing intensity. Without heat losses it presents three successive regimes easily distinguishable in the log-log representation: i) a regime of semi-infinite medium producing a straight line with a $-1 / 2$ slope, the position of which is depending on the sole effusivity of the material, ii) a transition zone mainly influenced by the diffusivity of the material and the thickness of the coupon (which is generally supposed known), iii) a final plateau is the sample is adiabatic and which depends on the volume heat capacity. If heat losses occur the thermogram changes : the first zone remains straight but the slope slightly decreases, the second one presents a negative curvature instead of a positive one, and the third one disappear. For the rear-face the asymptotic plateau is replaced by a maximum.

Without heat losses, the basic and simpler way to identify the diffusivity consists for the front-face configuration to measure the time of occurrence of the intersection of the two asymptotes which correspond to the Fourier value of $1 / \pi$ $=0.3183$, and for the rear-face to measure the half rise time which corresponds to a value of 0.139 of the Fourier. Heat 
losses make disappear these two characteristic points and require to search for new ones, the positions of which will depend on the Biot number. This approach has been done since a long time for the rear-face technique [1-5]. It is the aim of the present study to make the same for the front-face technique.

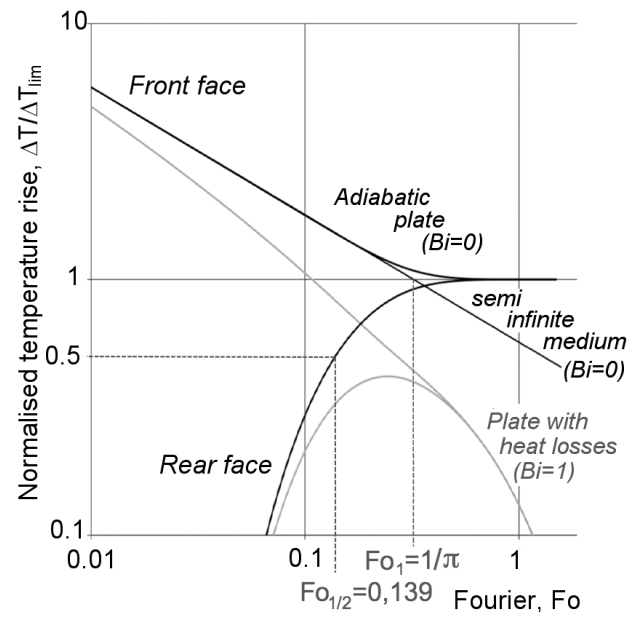

Fig. 1. Normalized pulsed thermograms of front- and rear-faces of a plate, with the characteristic points used for diffusivity identification in adiabatic conditions.

\section{In search of characteristic points for front-face thermograms}

To define new characteristic points where to proceed to the diffusivity identification, we can use the possibilities offered by the TSR method proposed at the beginning of the century by Shepard [9], in particular to consider the maximums of the first two logarithmic derivatives of the thermogram.

Let us recall that the TSR method consists to fit the pulse thermogram by logarithmic polynomials :

$$
\log _{10}(\Delta T)=a_{0}+a_{1} \log _{10}(t)+a_{2}\left[\log _{10}(t)\right]^{2} \ldots+a_{n}\left[\log _{10}(t)\right]^{n}
$$

and to obtain the logarithmic derivatives by derivations of the polynomial, which avoids to increase too much the noise. Until now the method is mainly used in a rather qualitative way for non destructive application, in particular because the images of the derivatives have a better quality than that of the temperature rises. Nevertheless, the TSR method can be used quantitatively for the identification of the diffusivity or the thickness of a structure, provided that one of the two be known [9].

The Figure 2 shows how the diffusivity can be identified from the following characteristic points, with and without heat losses. In the adiabatic case, the three remarkable points are the intersection of the asymptotes of the thermogram, the half-rise of the first derivative (value of 0.25 ) and the top of the second derivative. The Fourier number of these three

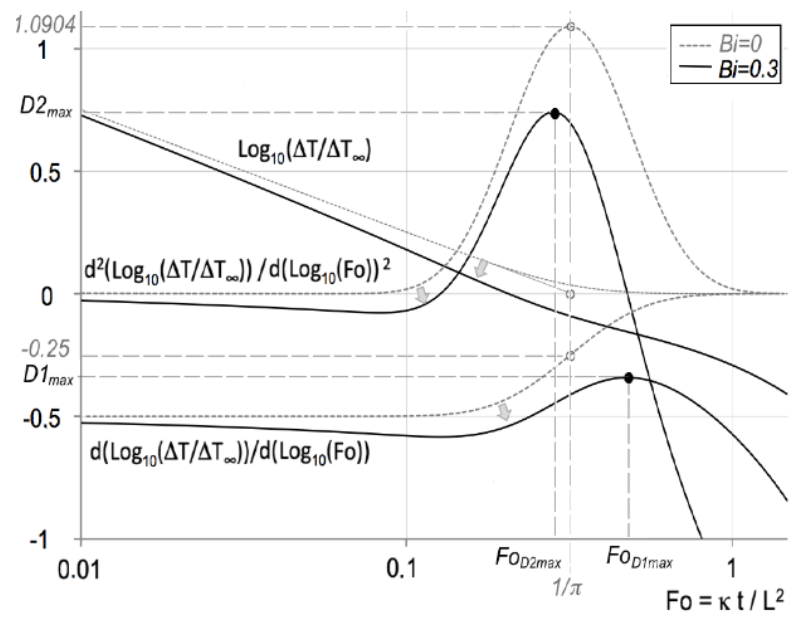

Fig. 2. Points usable to identify the diffusivity from the front-face pulsed heating measurement. Case of an adiabatic plate (Bi=0) taken from [10] and of one with heat losses (Bi=0.3). 
points is the same. In the non-adiabatic case, the direct exploitation of the thermogram is not possible, the half-rise of the first derivative becomes difficult to define and for high losses disappears, and the top of the second derivative still exists but has a lower value and occurs earlier. A second characteristic point can be the top of the first derivative. The value and time of occurrence of the first derivative, $D 1$, respectively $D 1_{\max }$ and $F_{O_{D 1} \max }$, and the ones of the second derivative, $D 2$, respectively $D 2_{\max }$, and $F O_{D 2 \max }$ can be considered for the identification.

Figure 3 shows the strong influence of the heat losses on the thermogram and its derivatives and the sensitivity of the parameters $D 1_{\max }, D 2_{\max }, F_{O_{D 1} \max }, F_{O_{D 2 \max }}$ to the Biot number. This sensitivity appears more clearly in Figure $3 d$, showing strong differences between each other. For both derivatives, the values of the maximums, $D 1_{\max }, D 2_{\max }$, are clearly more sensitive to the heat losses than the time of occurrence, $F_{O_{D 1} \text { max }}, F_{O_{D 1} \text { max }}$. The use of the peak of the $2^{\text {nd }}$ derivative a priori seems interesting since it occurs earlier than the one of the $1^{\text {st }}$ derivative, which leads to a better signal-to-noise ratio when considering real data. Conversely, the sensitivity to the Biot number of the time of occurrence of this derivative, $F_{O_{D 2 m a x}}$, appears very low as compared to the one of the other three parameters (see Figure $3 \mathrm{~d}$ ).

(a)
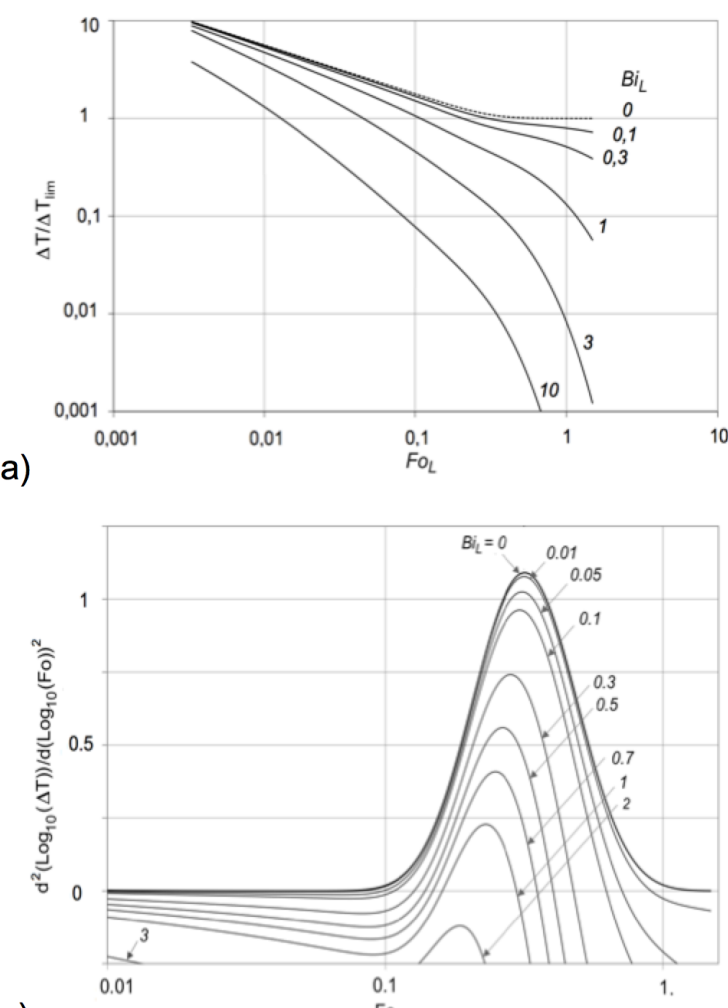

(c)

Fig. 3. Influence of heat losses (Biot number) on the front-face thermograms (a), the first logarithmic derivatives (b), the second one (c), and sensitivity to Biot number curves of the 4 parameters $D 1_{\max }, D 2_{\max }, F_{O_{D 1} \text { max }}, F_{O_{D 2 m a x}}(d)$.

\section{Identification of the diffusivity from the maximums of the first two derivatives}

\subsection{Description of the method}

\subsubsection{Using the first derivative}

The proposed identification method includes several steps:

- Thermogram fitting of thermogram $\log _{10}(\Delta T)=f\left(\log _{10}(t)\right)$ by a polynomial (TSR method);

- Derivatives of the polynomial (TSR method);

- Localisation of the maximum of the first derivative and evaluation of its value $D 1_{\max }$ and time of occurrence, $t_{D 1 \text { max }}$;

- Calculation of the theoretical Fourier number of the maximum of the derivative, $F O_{D 1}$ max , thanks to a correlation established from the simulation results (Figure $4 a$ ) between the experimental value of $D 1_{\max }$ and this Fourier number:

$$
F o_{D 1_{\max }}=-0.0798\left[\log _{10}\left(-D 1_{\max }\right)\right]^{3}-0.3047\left[\log _{10}\left(-D 1_{\max }\right)\right]^{2}-0.6999 \log _{10}\left(-D 1_{\max }\right)+0.2095
$$




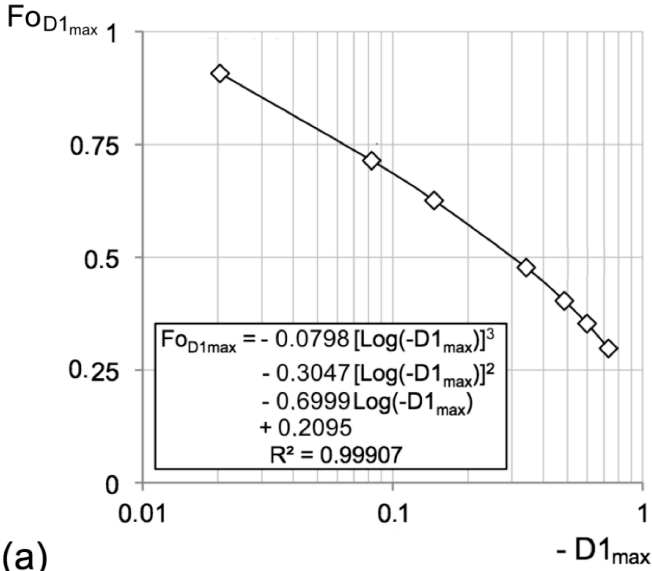

(a)

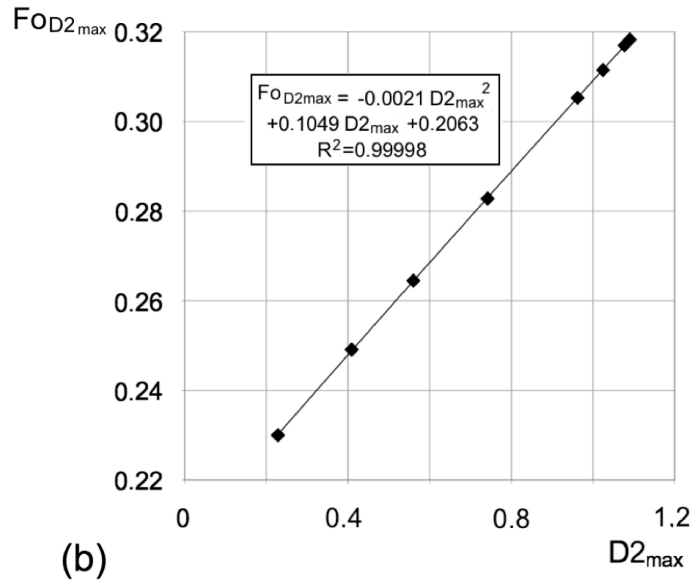

Fig 4. Relation between $F O_{D 1_{\max }}$ and $D 1_{\max }(\mathrm{a})$; ditto for $F O_{D 2_{\max }}$ and $D 2_{\max }$ (b).

- The experimental value of $t_{D 1 \max }$ is put in the expression of $F_{O_{D 1} \text { max }}$ leading to the value of the $\kappa / L^{2}$ ratio:

$$
\kappa / L^{2}=F o_{D 1_{\max }} / t_{D 1_{\max }}
$$

- Supposing known one of the two parameters $\kappa$ and $L$, the second one is easily deduced:

$$
\kappa=L^{2} F O_{D 1_{\max }} / t_{D 1_{\max }} \text { or } L=\sqrt{\kappa t_{D 1_{\max }} / F O_{D 1_{\max }}}
$$

\subsubsection{Using the second derivative}

The same procedure can be used with the second derivative, using a relation between the Fourier of occurrence, $F O_{D 2_{\max }}$, and the value of its maximum, $D 2_{\max }$ (see Figure $4 \mathrm{~b}$ ):

$$
F O_{D 2_{\max }}=-0.0021\left(D 2_{\max }\right)^{2}+0.1049 D 2_{\max }+0.2063
$$

\subsection{Intrinsic accuracy of the method}

\subsubsection{Bias introduced by the TSR method and the use of Eqs. (2) and (5)}

The correlations (2) and (5) are established from thermograms calculated by a numerical finite difference method (Euler) validated by comparison to a semi analytical solution (Laplace transform). The discrepancies between the two approaches is always $<0.1 \%$. These thermograms simulate experiments without noise. Practically, experimental thermograms suffer from noise which is eliminated by the TSR method which fit them by a series of logarithmic polynomials. This operation introduces a bias to the identified diffusivity. So, the application of the method to the theoretical thermograms allows to estimate the intrinsic accuracy of the identification. Reference [10] demonstrated that without losses, the identification of diffusivity from a characteristic point of the logarithmic derivatives needs a judicious choice of the polynomial degree and of the temporal window on which operate the fitting. In the present case, due to the important changes in the shape of the thermograms and their derivatives, it is possible that the use of the Eqs. (2) and (5) and the choice of the aforementioned parameters lead to different results, which justifies to know the intrinsic accuracy for optimizing the procedure. For each case, corresponding to a given value of the Biot number, a given degree of the fitting polynomial, $n$, and a given temporal window, we have calculated the fitted thermogram and its derivatives and applied the identification procedure allowing to estimate the diffusivity from the knowledge of the value and the occurrence time of the derivative peaks.

\subsubsection{Influence of the choice of the time window}

Three windows have been tested: large, $F_{O} \in[0.01-1.5]$, medium, $F_{O} \in[0.04-1.5]$, and narrow, Fo $\in$ [0.1-1.3]. As seen in Table 1, which presents the accuracy on the identified Fourier number of occurrence of the first derivative peak, $F_{O_{D 1 \max }}$, the optimal choice for the temporal window, whatever be the degree of the fitting polynomial, appears similar to the one of the study presented in [10], that is the best choice corresponds to the narrow window, and the less relevant to 
Table 1. Combined influences for $B i=0.1$ of the choices of the time window and of the degree of the polynomials on the accuracy of the identified occurrence Fourier of the peak of the $1^{\text {st }}$ logarithmic derivative, Fo $O_{D 1 \max }$.

\begin{tabular}{|c|c|c|c|c|c|c|}
\cline { 2 - 6 } \multicolumn{1}{c|}{} & \multicolumn{5}{c|}{ Degree of the polynomial } & \multicolumn{1}{c}{} \\
\hline Window (Fourier) & 5 & 6 & 7 & 8 & 9 & Mean \\
\hline$[0.01-1.5]$ & $-3.25 \%$ & $0.60 \%$ & $0.69 \%$ & $1.77 \%$ & $1.03 \%$ & $1.47 \%$ \\
\hline$[0.04-1.5]$ & $1.20 \%$ & $0.58 \%$ & $1.25 \%$ & $0.48 \%$ & $0.55 \%$ & $0.81 \%$ \\
\hline$[0.1-1.3]$ & $0.21 \%$ & $0.83 \%$ & $0.31 \%$ & $0.29 \%$ & $0.2 \%$ & $0.37 \%$ \\
\hline
\end{tabular}

the larger window. This trend, obtained for $B i=0.1$, is verified for other values of $B i$. Consequently, it has been decided to consider just this narrow window for the rest of the theoretical study.

\subsubsection{Influence of the choice of the degree of the logarithmic polynomials}

The influence on the derivatives of the degree of the polynomials $(5 \leq n \leq 9)$ has been studied for the narrow window and Biot numbers between 0.01 to 1 . The results are presented in Figure 5 . The influence of the degree of the polynomials is essentially marked at the borders of the window. In the vicinity of the derivative peaks all the curves of the $1^{\text {st }}$ derivative are merged. On the contrary, for the $2^{\text {nd }}$ derivative, noticeable differences are noted. The relative errors on the diffusivity identified from the position (Fourier of occurrence and value) of the peaks of the derivatives are given in Table 2. In case of thickness measurement (diffusivity supposed known) these errors should be two times less (see relations (4)).
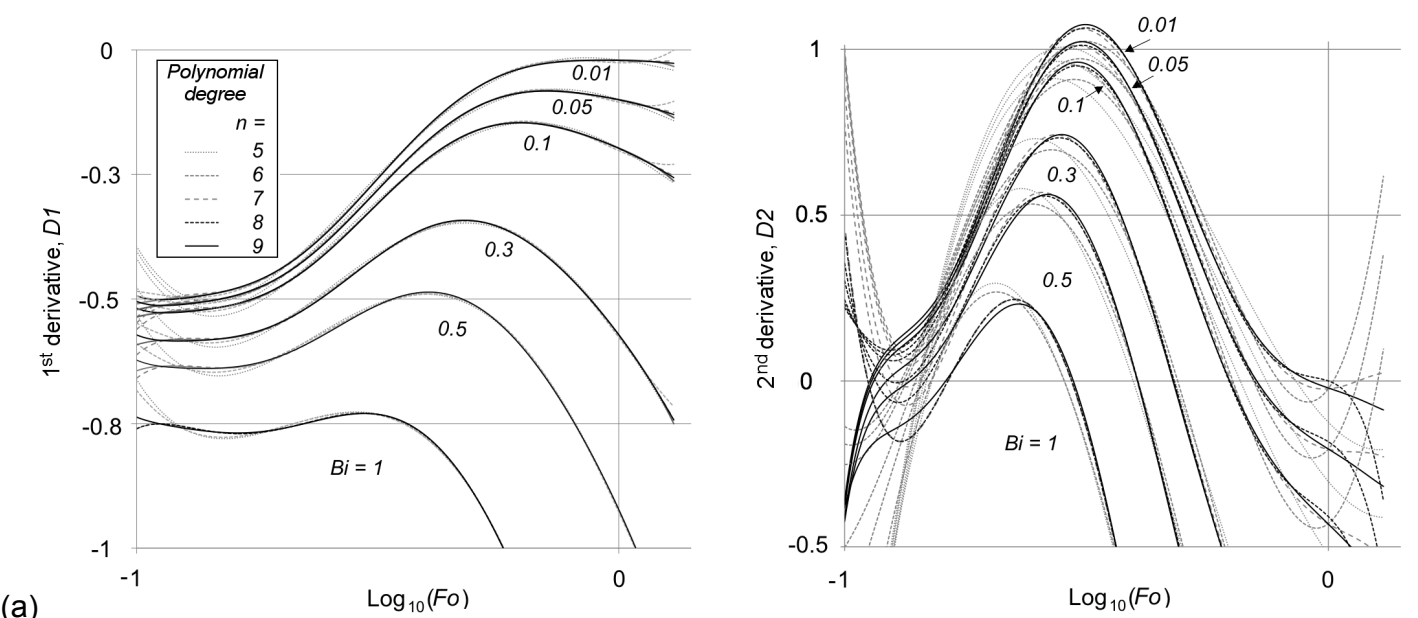

(b)

Fig. 5. Restitution of first (a) and second (b) derivatives of the thermograms by derivation of the logarithmic polynomials. Influence the degree of the polynomials. Results obtained for the narrow time window Fo $\in$ [0.1-1.3].

Table 2. Intrinsic accuracy of the diffusivity (a) identified from the peak of the $1^{\text {st }}$ derivative, $D 1_{\text {max }}$, (b) from the peak of the $2^{\text {nd }}$ derivative, $D 2_{\text {max }}$. Results for the narrow time window, Fo $\in[0.1-1.3]$.

\begin{tabular}{|c|ccccc|}
\cline { 2 - 6 } \multicolumn{1}{c|}{} & \multicolumn{5}{c|}{ Polynomial degree, $n$} \\
\hline Biot & 5 & 6 & 7 & 8 & 9 \\
\hline 0.01 & $10.8 \%$ & $14.3 \%$ & $5.0 \%$ & $9.4 \%$ & $1.0 \%$ \\
0.05 & $3.6 \%$ & $2.4 \%$ & $0.5 \%$ & $1.2 \%$ & $0.1 \%$ \\
0.10 & $4.5 \%$ & $0.6 \%$ & $0.3 \%$ & $0.8 \%$ & $0.4 \%$ \\
0.30 & $1.4 \%$ & $2.4 \%$ & $0.5 \%$ & $0.5 \%$ & $0.1 \%$ \\
0.50 & $1.2 \%$ & $1.3 \%$ & $0.3 \%$ & $0.7 \%$ & $0.4 \%$ \\
1.00 & $4.6 \%$ & $4.5 \%$ & $1.3 \%$ & $1.3 \%$ & $0.7 \%$ \\
\hline Mean & $4.3 \%$ & $4.2 \%$ & $1.3 \%$ & $2.3 \%$ & $0.4 \%$ \\
\hline$\sigma$ & $1.7 \%$ & $1.6 \%$ & $0.9 \%$ & $1.3 \%$ & $0.3 \%$ \\
\hline
\end{tabular}

(a) Using the 1st derivate, D1

\begin{tabular}{|c|c|}
\hline Mean & $\sigma$ \\
\hline $8.11 \%$ & $5.18 \%$ \\
\hline $1.54 \%$ & $1.44 \%$ \\
$1.30 \%$ & $1.80 \%$ \\
$0.96 \%$ & $0.94 \%$ \\
$0.79 \%$ & $0.42 \%$ \\
$2.49 \%$ & $1.91 \%$ \\
\hline
\end{tabular}

$|\Delta \kappa| / \kappa \quad \geq 10 \%$

\begin{tabular}{|c|ccccc||c||c|}
\cline { 2 - 6 } \multicolumn{1}{c|}{} & \multicolumn{5}{c|}{ Polynomial degree, $n$} & \multicolumn{1}{c|}{} \\
\hline Biot & 5 & 6 & 7 & 8 & 9 & Mean & $\sigma$ \\
\hline 0.01 & $9.1 \%$ & $0.7 \%$ & $2.3 \%$ & $0.1 \%$ & $0.6 \%$ & $2.5 \%$ & $3.74 \%$ \\
0.05 & $10.1 \%$ & $0.1 \%$ & $2.6 \%$ & $0.3 \%$ & $0.8 \%$ & $2.8 \%$ & $4.22 \%$ \\
0.10 & $11.2 \%$ & $0.7 \%$ & $2,9 \%$ & $0.6 \%$ & $0.8 \%$ & $3.2 \%$ & $4.57 \%$ \\
0.30 & $13.7 \%$ & $4.0 \%$ & $3.4 \%$ & $1.5 \%$ & $0.8 \%$ & $4.7 \%$ & $5.21 \%$ \\
0.50 & $14.9 \%$ & $7,2 \%$ & $3.6 \%$ & $2.3 \%$ & $0.9 \%$ & $5.8 \%$ & $5.59 \%$ \\
1.00 & $11.5 \%$ & $10.9 \%$ & $2.7 \%$ & $2.7 \%$ & $0.6 \%$ & $5.7 \%$ & $5.10 \%$ \\
\hline Mean & $11.7 \%$ & $3.9 \%$ & $2.9 \%$ & $1.2 \%$ & $0.8 \%$ & & \\
\hline$\sigma$ & $4.5 \%$ & $1.5 \%$ & $1.1 \%$ & $0.4 \%$ & $0.1 \%$ & & \\
\hline
\end{tabular}

(b) Using the 2nd derivate, D2 
The main conclusions deduced from Table 2 are the followings: i) Higher the degree $n$, better the accuracy of the identified diffusivity, ii) The use of the degree 9 is recommended since accuracies better than $1 \%$ are obtained for the explored Biot domain, iii) The use of polynomials of degree smaller or equal to 6 are to be avoided, iv) For low Biot numbers $(B i \leq 0.05)$ and $n \geq 6$ the use of the second derivative has to preferred, $v)$ on the contrary for large and medium Biot numbers $(B i \geq 0.05)$ the use of the first derivative is recommended.

The high intrinsic accuracy obtained with $n=9$ is comparable to that of rear-face pulsed techniques for diffusivity measurements, for which an effective accuracy of $3 \%$ is difficult to reach. In practice, to the intrinsic errors, errors due to the noise and low frequency drifts of the camera must be added. The latest become more important with the derivations of the thermograms. All these conclusions have to be faced to the results of real experiments.

\section{Experimental validation}

The simulations of the previous Section show that the domain of application of the method corresponds to Biot numbers between a few hundredths and unity. So, it is possible to define the materials and thicknesses for which the method will be useful. Figure 6 presents the domain in the space (thickness - thermal conductivity) and mentions the corresponding types of materials, from super-insulators to metals, and thicknesses. The highly shaded domain is the one for which the method is not adapted, and the lightly shaded the one for which the method leads to accurate measurements in normal ambient conditions. The present approach expands the domain of application by a factor of 100 as concerns the thickness of the tested structures and for a given thickness allows to make measurement on materials 100 times less conductors.

The method has been validated experimentally by measuring the diffusivity of a $5.24 \mathrm{~mm}$-thick plate made of carbon-epoxy used in previous NDE studies [13-15]. Both rear- and front-face measurements are made with the same IR camera (FLIR X6540sc), working in short waves [1.5 - 5] $\mu \mathrm{m}$. NETD is $20 \mathrm{mK}$ and space resolution $640 \times 512$ pixels. Two flash lamps Elinchrom deliver a total pulse energy of $6 \mathrm{~kJ}$ during $4 \mathrm{~ms}$. The frame rate is $100 \mathrm{~Hz}$. The thermograms are obtained by calculating the mean of $30 \times 30$ pixel zones.

Supposing a thermal conductivity of the plate of $0.6 \mathrm{Wm}^{-1} \mathrm{~K}^{-1}$, a usual value for this type of material, the experimental conditions are plotted on the graph of Figure 6. They shows that the tests is in the central part of the application domain of the method and correspond to a Biot near of 0.1 . This is verified by the thermograms presented in Figure $7 \mathrm{~b}$, which does not present any final plateau.

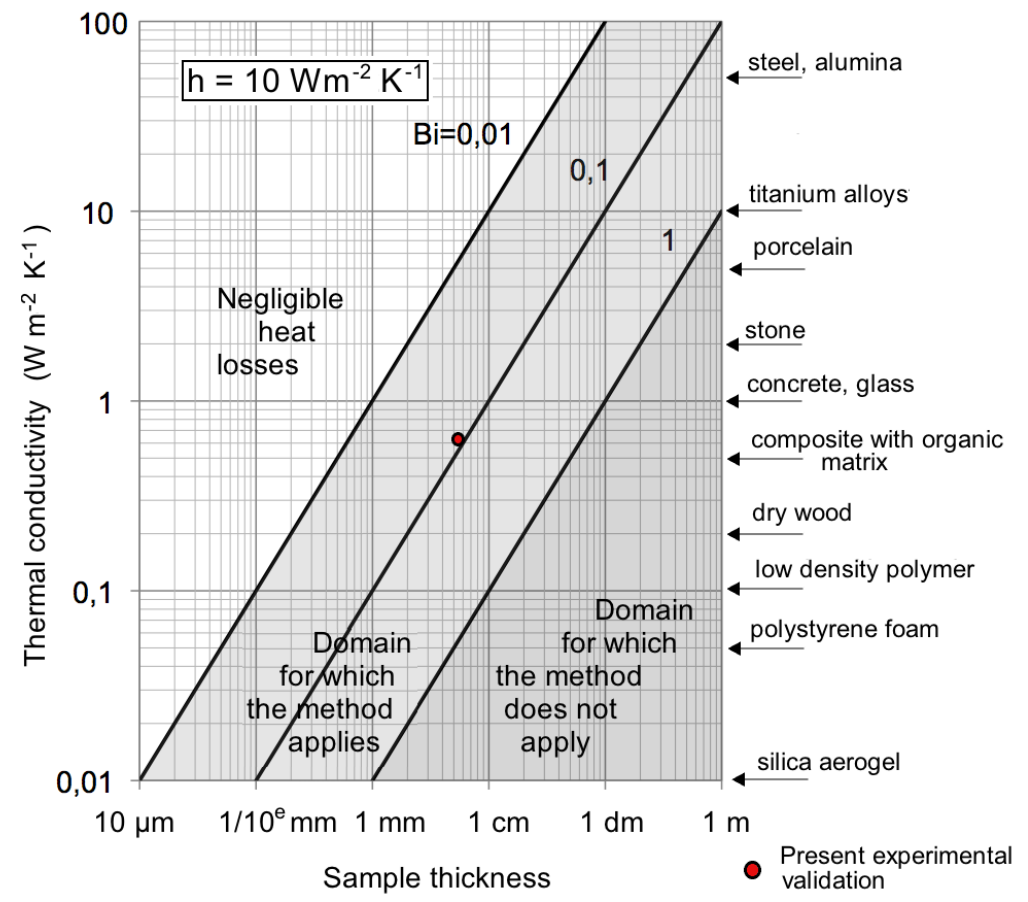

Figure 6. Domain of application of the method in normal ambient temperature and pressure conditions $\left(h=10 \mathrm{Wm}^{-2} \mathrm{~K}^{-1}\right)$. The experimental conditions of the tests presented in Section 5.2 are presented as well.

\subsection{Rear-face diffusivity measurement}

To validate the present method we chose the rear-face flash diffusivity method taken into account the heat losses. Among the various data processing methods we chose for its simplicity and accuracy the partial times method proposed by Degiovanni in Reference [5]. It considers five characteristics points of the thermogram corresponding to the normalized temperature rises $\Delta T / \Delta T_{\max }=1 / 3,1 / 2,2 / 3,5 / 6$ and 1 . The following three identification formulas: 

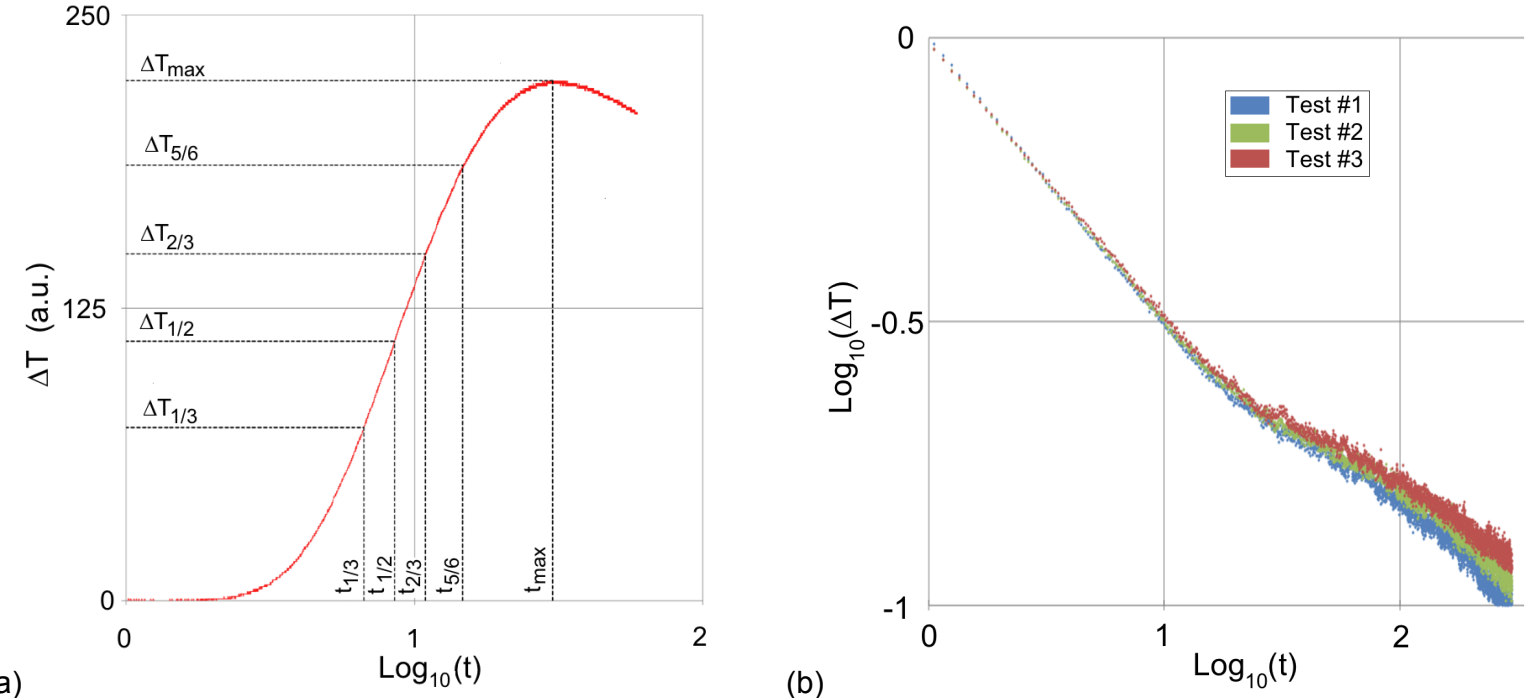

Fig. 7. Validation of the method by comparison to a rear-face measurement. (a) Experimental rear-face thermogram and diffusivity evaluation by the partial times method; (b) Front-face thermograms (normalized temperature rise).

$$
\begin{aligned}
& \kappa_{1 / 3}=\left[0.818-1.708\left(t_{1 / 3} / t_{5 / 6}\right)+0.885\left(t_{1 / 3} / t_{5 / 6}\right)^{2}\right] L^{2} / t_{5 / 6} \\
& \kappa_{1 / 2}=\left[0.954-1.581\left(t_{1 / 2} / t_{5 / 6}\right)+0.558\left(t_{1 / 2} / t_{5 / 6}\right)^{2}\right] L^{2} / t_{5 / 6} \\
& \kappa_{2 / 3}=\left[1.131-1.222\left(t_{2 / 3} / t_{5 / 6}\right)\right] L^{2} / t_{5 / 6}
\end{aligned}
$$

use the times of occurrence $t_{1 / 3}, t_{1 / 2}, t_{2 / 3}, t_{5 / 6}$ (see Figure $7 \mathrm{a}$ ). The three estimates of the diffusivity are very similar, leading to a mean value of $4.1710^{-7} \mathrm{~m}^{2} \mathrm{~s}^{-1}\left(\sigma=0.0210^{-7} \mathrm{~m}^{2} \mathrm{~s}^{-1}\right)$. As a reference for the material diffusivity, we will chose the ealier identified value, $4.1910^{-7} \mathrm{~m}^{2} \mathrm{~s}^{-1}$, rounded up to the value of $4.2,10^{-7} \mathrm{~m}^{2} \mathrm{~s}^{-1}$.

\subsection{Front-face diffusivity measurement}

Several tests were done on the plate in a sound zone, with a duration of $600 \mathrm{~s}$ each, comprising a $300 \mathrm{~s}$ preliminary plateau to define the baseline, followed by a $300 \mathrm{~s}$ - acquisition of the temperature rise created by the pulse heating. The initial plateau allowed to measure a mean drift of $0.20 \mathrm{mK}^{-1} \mathrm{~s}^{-}$and to correct the thermogram. Three tests took place the same day: one in the morning (test \#1), a second one at noon (test \#2) and the last one (test \#3) in the afternoon. All thermograms have been normalized by the temperature rise at $t=1 \mathrm{~s}$ (Figure 7b). The heat losses effects are important and totally suppress the final plateau. The signal-to-noise ratio, especially at the end of the tests, is clearly lower than that of the rear-face thermogram. The noise itself, estimated from the initial plateau is equal to $19 \mathrm{mK}$, which is in agreement with the nominal performances of the camera.

The thermogram shown in Figure 8 is the initial stage of test \#1 after subtraction of the mean value and correction of the mean drift. It demonstrates that beside a high frequency noise, low frequency fluctuations of the order of tens of millihertz exist. Such fluctuations are able to deeply disturb the derivatives of the thermogram, leading to degrade the accuracy of the diffusivity identification.

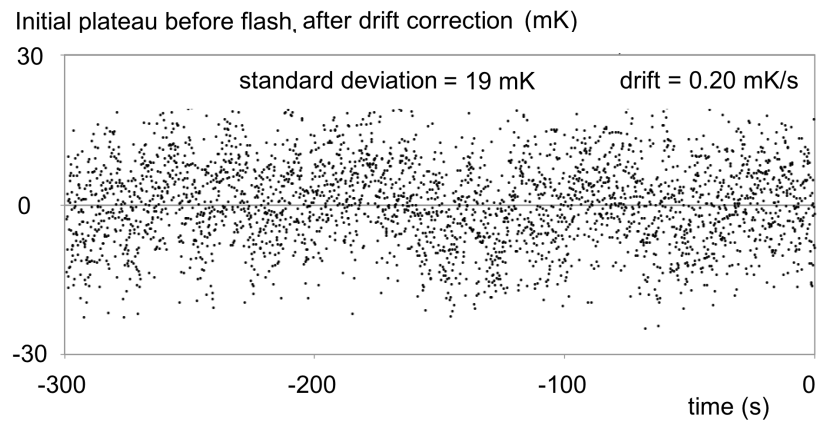

Fig. 8. Test \#1, initial plateau before flash, after linear drift correction, showing low frequency fluctuations. 
Time window for fitting: $[1 \mathrm{~s}-300 \mathrm{~s}]$
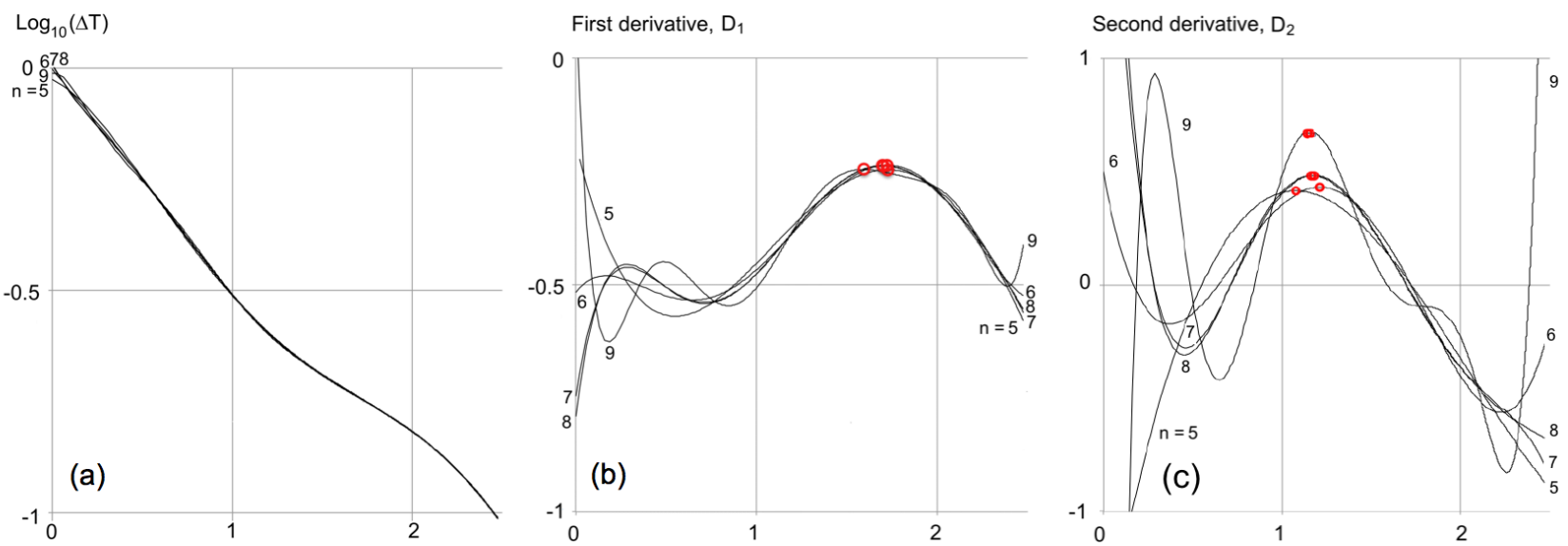

Time window for fitting: $[5 \mathrm{~s}-200 \mathrm{~s}]$
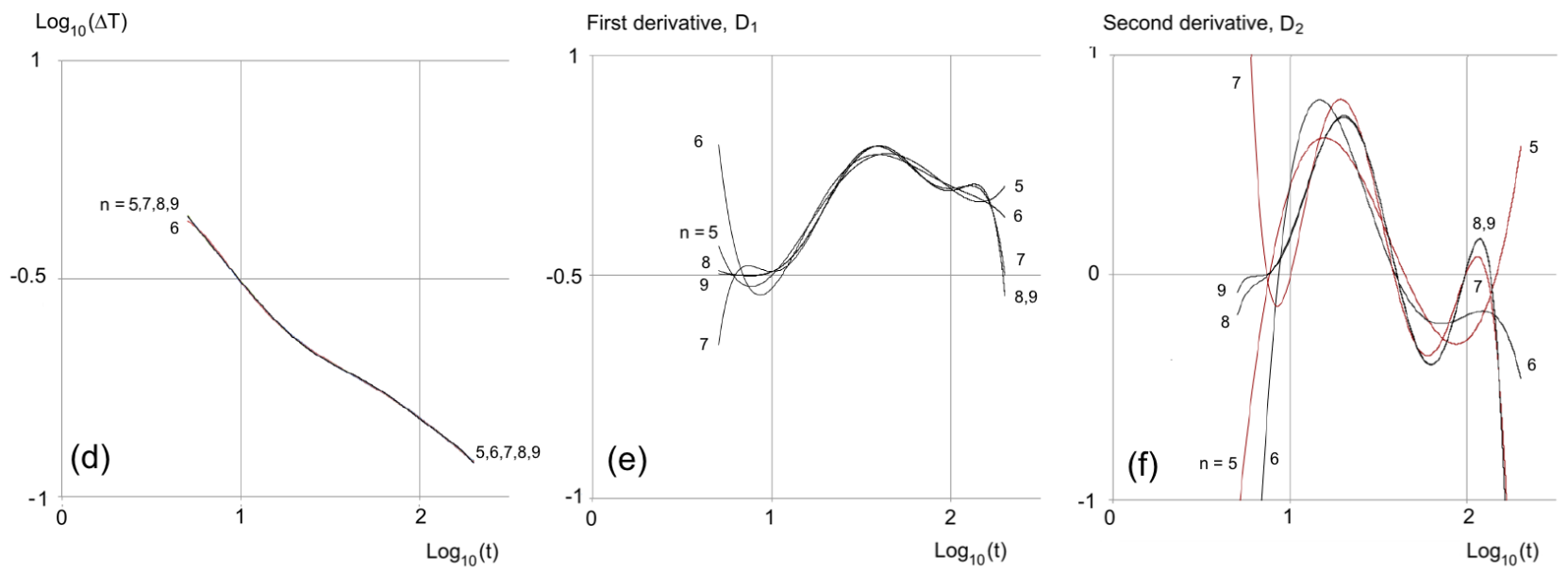

Fig. 9. Combined influence of the time-window ([1s-300s] and [5s-200s]) and of the polynomial degree $(n=5$ to 9$)$ on: $(a, d)$ the fitted thermograms $\log _{10}(\Delta T)=f\left(\log _{10}(t),(b, e)\right.$ their first derivatives $\left(D_{1}\right),(c, f)$ their second derivative $\left(D_{2}\right)$. The thermogram is normalized by its value at time $t=1 \mathrm{~s}$. Test \#1.

Figure 9 presents the combined influence of the time window and polynomial degree on the reconstruction of the experimental thermogram (Test \#1) and of its first two logarithmic derivatives. Both parameters have a noticeable influence on the amplitude and time of occurrence of the the peaks of the derivatives, and consequently on the accuracy of the identified diffusivity.

Table 3 presents the relative errors on the diffusivities deduced from the three tests. These errors have been calculated supposing exact the value found from the rear-face test $\left(\kappa=4.210^{-7} \mathrm{~m}^{2} \mathrm{~s}^{-1}\right)$. The influence of the following three parameters are detailed: i) degree of the polynomials used by the TSR method $(5 \leq n \leq 9)$, ii) the time window considered for the polynomial fitting (a narrow window, [5-200] s, and wider window, [1 - 300] s, iii) the choice of the peak derivatives $\left(D 1_{\max }\right.$ and $\left.D 2_{\text {max }}\right)$.

Table 3 shows that: i) the errors are more important than the intrinsic errors (Table 2), which was foreseen since there are noise and fluctuations in the experiments, not considered in the simulations; ii) the three tests present noticeable disparities; iii) the use of the $1^{\text {st }}$ derivative generally leads to poorer results than the ones of the $2^{\text {nd }}$ derivative; iv) the variations of the accuracy induced by changing the polynomial degree, although a little chaotic, present an improvement when passing from 5 to $9 ; \mathrm{v}$ ) the improvement of the accuracy verified with the simulations when passing from a large window to a narrow one is not systematic.

The right part of Table 3 presents the mean values and the standard deviations of the identified diffusivities considering the three tests. Some very important errors make the reader perplexed regarding the strategy to follow to guaranty an accurate measurement. The solution could be the following: lest us come back to simple principles of thermophysics: 
Table 3 . Comparative study of the influence of the degree of the polynomial, the temporal window considered for the regression and the derivative (D1 or D2) on the accuracy of the diffusivity identified from the three tests.

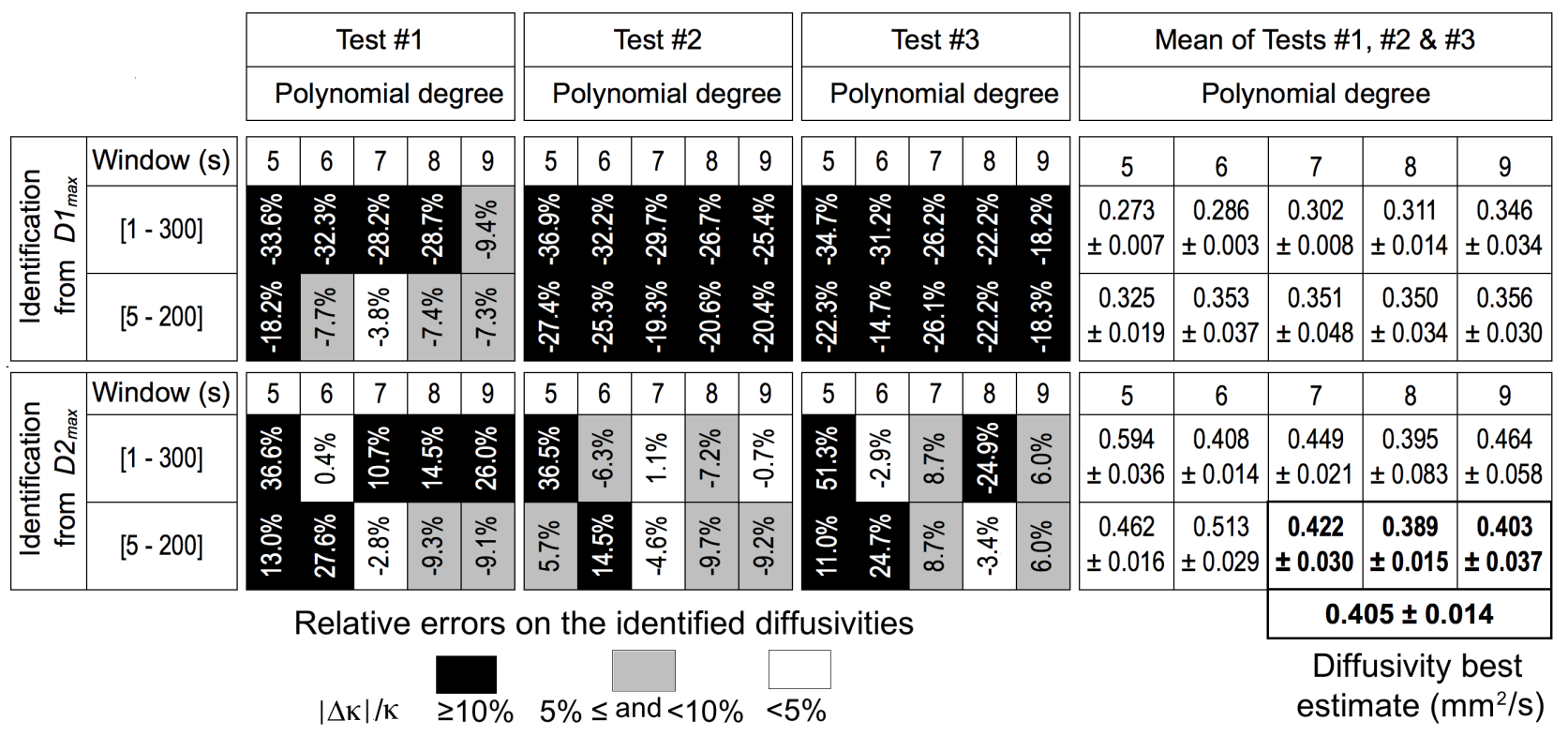

- Favour given to earlier identification, so preference given to the use of $D 2_{\max }$ instead of $D 1_{\max }$ (see [16], pp. 23-24);

- Choice of a narrow time window for the polynomial fitting, with a size of the order of one decade and a very small part given to the semi infinite medium regime (straight line part in log-log representation);

- No use of low degree for the polynomials ( $n=5$ et 6 not considered);

- Realization of several tests and consideration of the mean value resulting from them.

Applying these rules leads to just consider in Table 3 the three values written in bold type and more particularly their mean value over the full trio: $\kappa=(4.05 \pm 0.01) 10^{-7} \mathrm{~m}^{2} \mathrm{~s}^{-1}$. This value is very near from that found from the rear-face technique, $\kappa=(4.17 \pm 0.02) \cdot 10^{-7} \mathrm{~m}^{2} \mathrm{~s}^{-1}$, which confirms the pertinence and efficiency of the criteria proposed. Nevertheless, these numbers must be considered with caution, since even for the very secure rear-face flash method of Parker, round-robin tests conducted with well-established laboratories (see for instance [17]), on reference homogenous materials, it was concluded that accuracies better than $3 \%$ were difficult to reach.

\section{Conclusions}

The present study demonstrates the possibility for front-face pulsed-stimulated thermography to measure the diffusivity of a structure even in presence of noticeable heat losses impeding to use classical data processing. The method is based on the TSR method and achieves the identification from the measured characteristics (amplitude and time of occurrence) of the peak of the first or second logarithmic derivatives. The method is based on two original correlations relating the normalized time of occurrence and the amplitude of the peaks - equations (2) and (5) -.

Numerical simulations have established that the intrinsic accuracy of the method is governed by three parameters: degree of the TSR polynomials, extension of the time window considered for the polynomial fitting of the thermogram, and choice of the derivative $\left(1^{\text {st }}\right.$ or $2^{\text {nd }}$ derivatives). The intrinsic accuracy is found excellent (less $1 \%$ error on the diffusivity) for a judicious choice of this parameters.

An experimental validation in presence of real noise and fluctuations of the IR camera signal has been done by application to a carbon-epoxy plate of $5.25 \mathrm{~mm}$ thickness. From the time evolution of the temperature rise during the $300 \mathrm{~s}$ following the pulse, diffusivity estimation have been obtained. Depending on the values of the aforementioned parameters the results present chaotic errors, generally much more important than the intrinsic errors. Nevertheless, applying a few rules based on some simple principles of thermophysics, it is possible to find results in good agreement with diffusivity measurements by a classical rear-face pulse heating technique.

An important lesson learned from the study could be the following: to use the logarithmic derivatives of a pulsed thermogram (TSR method) with metrological ambitions is difficult. This lack of realism leads sometimes people to propose to exploit even the second peak of the derivatives, a situation even more difficult when dealing with experimental data. In fact, this is probably due to the fact that most of the applications of TSR are in the field of END for which a very high accuracy is not necessary.

Let us mention that the experimental case here presented is difficult considering the large duration of the test. Other conditions, needing shorter experiments to produce heat losses effects may be encountered. They should lead to an easier application of the method. Finally, to correct efficiently the thermogram for such very long test durations, a solution could be to acquire in real time the fluctuations of the camera signal in a zone of the images where no stimulation occurred. 


\section{REFERENCES}

[1] Heckman RC. Finite pulse-time and heat-loss effects in pulse thermal diffusivity measurements. J Appl Phys. 1973;44(4):1465-1470.

[2] Clark III LM, Taylor RE. Radiation loss in the flash method for thermal diffusivity. J Appl Phys. 1975;46(2): 714719.

[3] Degiovanni A. Diffusivité et méthode flash. Rev Gén Therm. 1977;185:420-442.

[4] Balageas DL. Nouvelle méthode d'interprétation des thermogrammes pour la détermination de la diffusivité thermique par la méthode impulsionnelle (méthode flash). Rev Phys Appl. 1982;17(4):227-237.

[5] Degiovanni A., Laurent M. Une nouvelle technique d'identification de la diffusivité thermique pour la méthode "flash". Rev Phys. Appl. 1986;21:229-237.

[6] Pech-May NW, Mendioroz A, Agustín Salazar A. Generalizing the flash technique in the front-face configuration to measure the thermal diffusivity of semitransparent solids. Rev Scien Instr. 2014;85:104902-1-6.

[7] Salazar A, Mendioroz A, Apiñaniz E, Pradere C, Noël F, Batsale J-C. Extending the flash method to measure the thermal diffusivity of semitransparent solids. Meas Sci Technol. 2014;25:035604.

[8] Krapez J-C, Hay B, Demange D, Gardette G, Levesque P, Passilly F. Méthode flash en face avant Optimisation de l'expérience pour un monocouche et un bicouche. Congrès de la Société Françaide des Thermiciens (SFT 2002), Vittel, France, 3-6 juin 2002.

[9] Shepard SM, Ahmed T, Rubadeux BA, Wang D, Lhota JR. Synthetic processing of pulsed thermographic data for inspection of turbine components. Insight. 2001;43(9):587-589.

[10] Balageas D. Thickness or diffusivity measurements from front-face flash experiments using the TSR (thermographic signal reconstruction) approach. Conférence QIRT 2010, Quebec, Canada, 27-30 Juill. 2010. Papier QIRT 2010-011. Available on QIRT Open Archives: http://dx.doi.org/10.21611/qirt.2010.011

[11] Balageas D, Boscher D. L'évaluation des pertes dans les expériences photothermiques impulsionnelles. Application à la détermination des coefficients de transfert convectif sur des maquettes en soufflerie. CR Acad Sci Paris. 1987; vol. 305, Série II:13-16.

[12] Balageas DL, Déom AA, Boscher, DM. Characterization and non destructive testing of Carbon-epoxy composites by a pulsed photothermal method. Mat Eval. 1987;45(4):461-465.

[13] Roche J-M, Balageas DL. Common tools for quantitative pulse and step-heating thermography - part II: experimental investigation. Quant Infrared Thermogr J. 2015;12(1):1-23.

[14] Roche J-M, Passilly F, Balageas D. A TSR-based quantitative processing procedure to synthesize thermal Dscans of real-life damage in composite structures. J Nondestruct Eval. 2015; 34:41.

[15] Balageas DL, Roche J-M, Leroy F-H.Comparative assessment of thermal NDT data processing techniques for carbon fiber reinforced polymers. Mat. Eval. 2017;75(8):1019-1031.

[16] Balageas DL. Defense and illustration of time-resolved pulsed thermography for NDE. Quant. Infrared Thermogr J. 2012;9(1):3-32.

[17] Hay B., Filtz JR, Hameury J., Rongione L. Uncertainty of thermal diffusivity measurements by laser flash method. Int. J. Thermophysics. 2005;26(6):1883. 